\title{
Contact network topology in tapped granular media
}

\author{
Roberto Arévalo \\ CNR-SPIN, Dipartimento di Scienze Fisiche, Universitá di Napoli “Federico II,” Naples, Italy \\ Luis A. Pugnaloni \\ Departamento de Ingeniería Mecánica, Facultad Regional La Plata, Universidad Tecnológica Nacional, \\ Avenida 60 esquina 124 s/n, 1900 La Plata, Argentina \\ Iker Zuriguel and Diego Maza \\ Departamento de Física, Facultad de Ciencias, Universidad de Navarra, E-31080 Pamplona, Spain
}

(Received 16 October 2012; published 8 February 2013)

\begin{abstract}
We analyze the contact network of simulated two-dimensional granular packings in different states of mechanical equilibrium obtained by tapping. We show that topological descriptors of the contact network allow one to distinguish steady states of the same mean density obtained with different tap intensities. These equal-density states were recently proven to be distinguishable through the mean force moment tensor. In contrast, geometrical descriptors, such as radial distribution functions, bond order parameters, and Voronoi cell distributions, can hardly discriminate among these states. We find that small-order loops of contacts-the polygons of the network-are especially sensitive probes for the contact structure.
\end{abstract}

DOI: 10.1103/PhysRevE.87.022203

PACS number(s): 81.05.Rm, 61.43.-j

\section{INTRODUCTION}

Dense granular media in mechanical equilibrium constitute a class of systems of deceptive simplicity. A paradigmatic example of such systems is a bed of grains inside a container which is subjected to vertical pulsed agitation, allowing the grains to relax between successive excitations. By setting the dimensionless vertical acceleration $\Gamma$ of the shaking, the system can explore a variety of configurations of mechanical equilibrium and reach a final steady state. This situation has been widely studied; experimentally [1-4], by means of simulations [5-7], and theoretically [8]. Ideally, we would like to understand the macroscopic or collective properties of the steady state of the material in terms of an ensemble of microscopic states (configurations), in analogy with the way in which we understand thermodynamics in terms of statistical mechanics.

A statistical description of this type was put forward by Edwards and Oakeshott [8] using solely the number of grains $N$ and the volume $V$ of the pack (or, equivalently, the packing fraction $\phi$ ) to characterize the microscopic states. This framework has been developed by several authors [9-12] over the years and has been shown to be useful to explain distinctive features of granular media. Despite the functionality of this idea, it has been recently suggested that the so-called $N V$ ensemble should be extended to take into account the stress propagation in the system to achieve a complete description of a granular packing [13-18].

On the other hand, recent experiments and simulations $[6,19]$ have shown that the packing fraction $\phi$ is not a monotonic function of the excitation intensity. Instead, a minimum packing fraction $\phi_{\min }$ is achieved at a certain value $\Gamma_{\min }$. Beyond this value the packing fraction increases again. It has been shown [20] that this behavior is observed in both in two and three dimensions using hard sphere models with various simulation techniques and soft frictional spheres simulated by discrete-element methods. This nonmonotonic behavior implies that steady states with equal average packing fractions can be reached with very different tap intensities. Then the question of whether or not these states are statistically equivalent naturally arises. This question has been thoroughly studied by means of simulations in Refs. [6,7] where it is found that a measure of the stress inside the system - the force moment tensor $\Sigma$-increases monotonically for the whole range of intensities considered. Hence, it is concluded that states at the two sides of the minimum are not equivalent, and that $\Sigma$ is a suitable candidate to complete the description of the equilibrium magnitudes that represent a granular system at the macroscopic level.

Provided that $\Sigma$ is shown to distinguish steady states of equal $\phi$, one would like to explore what other descriptors of the granular packing would display such sensitivity to changes in the configurations which are not discernible by $\phi$. Since the external stress applied to the system is equilibrated exclusively by contacts, it seems reasonable that the properties of the contact network may carry some of the key information contained in $\Sigma$. We can then use the concepts and tools of complex networks [21] to analyze the properties of the contact structure. This will be used to evaluate whether there is any network property that allows a clear distinction between states with the same $\phi$. At this point, let us note that any static granular system can be considered in terms of nodes (the grains) and edges (the contacts between grains). This approach has been recently applied to address a wide variety of granular phenomena such as tapping [22], porosity [23], force distribution [24], rheology [25], signal propagation [26], and the jamming transition [27].

The aim of the present work is to characterize the contact networks of the different steady states of packings generated by tapping. On the one hand, we will show that it is not possible to clearly uncover the differences between steady 
states of equal packing fraction using standard geometrical characterizations such as the pair correlation function and Voronoi cell distribution. Nevertheless, we will demonstrate that states with the same volume obtained with different tap intensities exhibit discernible contact structures, rendering them nonequivalent.

\section{SIMULATION AND TAPPING PROTOCOL}

We use soft-particle molecular dynamics simulations in two dimensions (2D), in which static friction is implemented through the usual Cundall-Strack model [28]. The details of the implementation have been described elsewhere [29], and in the following we give the values of the interaction parameters used in the present work. The friction coefficient is $\mu=0.5$, stiffness in the normal direction of the contact $k_{n}=10^{5}(\mathrm{mg} / \mathrm{d})$, damping parameter in the normal direction $\gamma_{n}=300(m \sqrt{g / d})$, stiffness in the tangential direction $k_{s}=\frac{2}{7} k_{n}$, and damping parameter in the tangential direction $\gamma_{s}=200(m \sqrt{g / d})$. The integration time step is set to $\delta=$ $10^{-4} \sqrt{d / g}$. The confining box (13.39d wide and infinitely high) contains $N=512$ monosized disks. Units are reduced with the diameter of the disks $d$, the disk mass $m$, and the acceleration of gravity $g$. Additional simulations for two different stiffnesses were run, with values $2 k_{n}$ and $k_{n} / 2$.

Tapping is simulated by moving the confining box in the vertical direction following a half sine wave trajectory $\{A \sin (2 \pi \nu t)[1-\Theta(2 \pi \nu t-\pi)]\}$. The excitation is applied with a constant frequency $v=\pi / 2(g / d)^{1 / 2}$, and hence it is solely controlled through the amplitude $A$. The tap is characterized by the dimensionless parameter $\Gamma=A(2 \pi \nu)^{2} / g$ although we would like to note that other parameters (like the dimensionless energy supplied to the system [30]) have been reported to be more suitable. In order to decide when the system has reached mechanical equilibrium, we implement a robust criterion based on the stability of particle contacts [29]. Then the packing fraction is calculated and a new tap is applied to the sample. The packing fraction was calculated in a slab of the bed that covers $50 \%$ of the height of the column and is centered on the center of mass of the system. Averages were taken over 500 taps (configurations) after we ensured that the system has reached a steady state for each value of $\Gamma$. In particular, we discard the 500 initial configurations in order to avoid any transient. By assuming that the values for the packing fraction obtained at each tap are normally distributed, we estimate the uncertainty of the mean packing fraction by calculating the upper and lower limits for a 95\% confidence interval (we will apply the same criterion for all the magnitudes introduced in the next sections). Using this protocol, we obtain the curve of the mean packing fraction $\phi$ in the steady state as a function of $\Gamma$ (Fig. 1). At low values of $\Gamma$, increasing the intensity of the taps leads to a decrease in the packing fraction. Then, beyond a certain value $\Gamma_{\min }$, this tendency is reversed and the density increases again up to values slightly below the ones obtained at low excitations. This qualitative behavior is independent of the frequency $v$ of the taps, although the position of the minimum is shifted to higher values of $\Gamma$ when $v$ is increased [7].

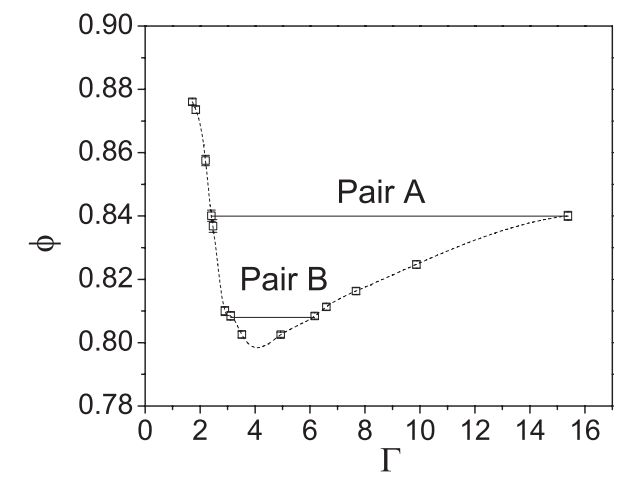

FIG. 1. Mean packing fraction $\phi$ of the steady states as a function of the tap intensity $\Gamma$. The horizontal lines join the states of same density to be compared in Secs. III and IV. The dashed line is a guide to the eye.

\section{GEOMETRICAL DESCRIPTORS}

It is usual to assume that the geometrical arrangement of the grains determines - at least at first order - the effective macroscopic properties of any heterogeneous material. However, the fact that different steady states may display the same values of packing fraction suggests the need for a quantification of the extent to which these states are truly different. One possibility to distinguish between states of equal packing fractions is to assess the density fluctuations. Although subtle differences were revealed in a previous work [7], these are difficult to observe unless a rather large collection of independent repetitions of the experiments is available.

In this section we explore whether there are other structural descriptors that can distinguish between two steady states of equal density in a clearer way than volume fluctuations. In this direction, it becomes natural to explore the pair correlation function [31] $g(r)$, corresponding to states of comparable packing fraction but obtained at very different tap amplitudes (Fig. 2). We choose a pair of states (indicated by horizontal lines in Fig. 1): one with a relatively high density (pair $A$, $\phi \simeq 0.84$ ) and the other with low density (pair $B, \phi \simeq 0.81$ ). In all cases, the correlation function presents well-defined peaks, which is a clear indication of the high degree of positional

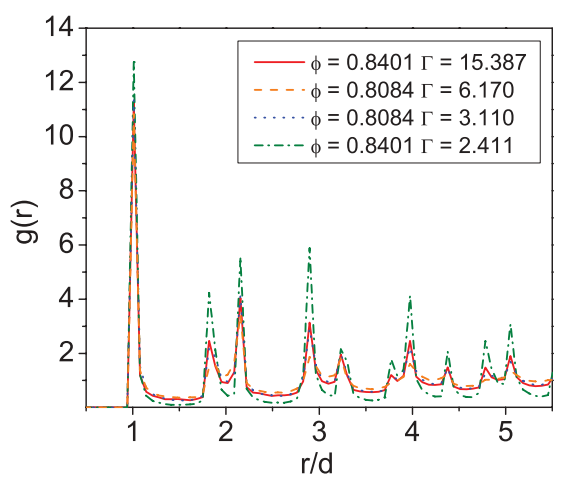

FIG. 2. (Color online) Pair correlation functions of the four states compared in the main text. The two states at higher density present sharper peaks, while the two states at lower density display more attenuated signals. However, it is not possible to distinguish between states of equal density. 


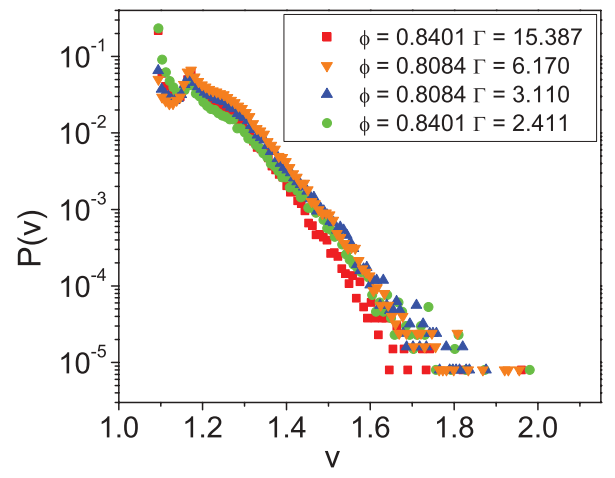

FIG. 3. (Color online) Size distribution of the area (normalized by the disk area) of the Voronoi cells for the four states compared in the text. All the distributions are very similar. It is possible to discriminate only states at high density from those at low density by looking at the height of the main peak, which is larger for the former.

ordering present in the system [32]. Indeed, the higher the packing fraction, the sharper the peaks in the distribution, which indicates an increase of the order in the sample as $\phi$ increases. In addition, when comparing the states with the same density, it is realized that the peaks are notably enhanced for the states obtained with lower $\Gamma$. This suggests a difference in the packing structure of states that display the same value of $\phi$. The combination of both effects (packing fraction and tap intensity) leads to a curious result: the $g(r)$ obtained for $\Gamma=15.34$ and $\Gamma=3.10$ are virtually indistinguishable although their packing fractions are different $(\phi \simeq 0.84$ and $\phi \simeq 0.81$ respectively).

As we use monosize particles, the Voronoi tessellation of the system obtained from the grain centers could be an alternative indicator of the degree of ordering displayed by the system at different tap amplitudes. This method has been used as a tool to assess the structure of granular materials, in both $2 \mathrm{D}$ and 3D $[3,33]$. In Fig. 3, we show the distribution of areas (normalized by the disk area) of the tessellations computed for the four states that we are comparing. In all cases, the decay is exponential for large areas and the curves show a high population of the minimum Voronoi cell at $v \sim 1.09$. This corresponds to the area of the Voronoi cell that is assigned to a disk in a perfect hexagonal packing of identical grains $v_{\text {hex }}=\frac{\sqrt{3}}{2} d^{2} \approx 1.0911$. The height of this peak allows for distinguishing between states of different density since high-density, ordered structures present a bigger number of hexagonal arrangements. However, the distributions for steady states of equal density are nearly indistinguishable.

Provided that the main differences in these indicators are usually related to the degree of hexagonal order and the existence of defects (localized or distributed) we considered the $Q_{l}$ order parameter proposed by Steinhardt et al. [34]. In particular, the value of $Q_{6}$ has been demonstrated to be a useful tool to describe order in hard-sphere systems [35]. Importantly, we will compute $Q_{6}$ in two different ways. First, as defined in Ref. [34] we consider as nearest neighbors any pair of particles that are closer than a distance 1.2d. Then we consider as nearest neighbors only those particles in contact. We denote by $Q_{6}$ the former quantity and $Q_{6}^{*}$ the latter. Notice that $Q_{6}$ reflects the geometrical order whereas $Q_{6}^{*}$ is related to
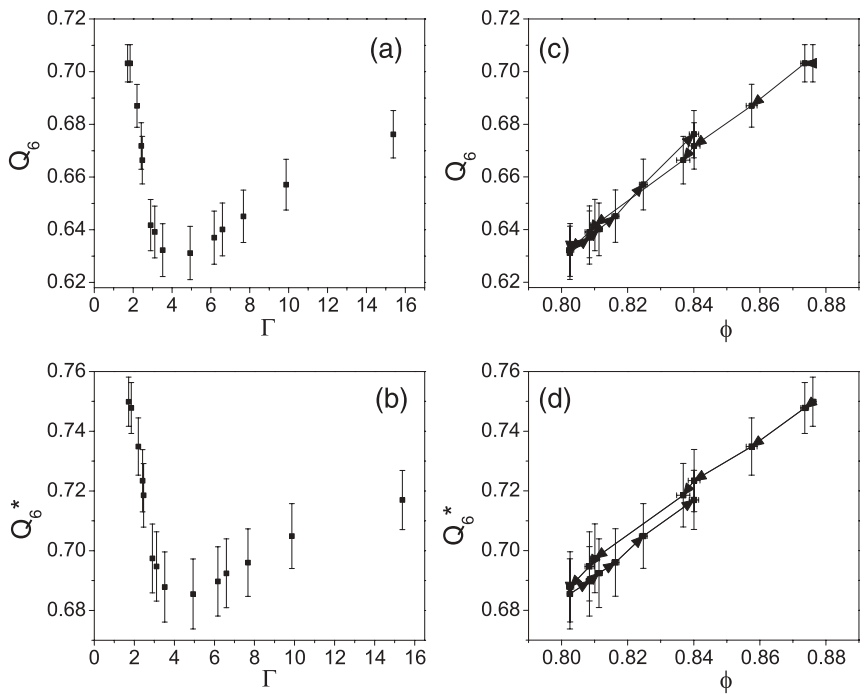

FIG. 4. Average value of the bond order parameter for nearest neighbors $Q_{6}$, upper panels, and contacts $Q_{6}^{*}$, lower panels. (a) and (b) show the mean value of the bond order parameter as a function of the tap amplitude $\Gamma$, and (c) and (d) as a function of the density $\phi$. In (c) and (d), the arrows indicate the direction of increasing $\Gamma$.

properties of the contact network. In Figs. 4(a) and 4(b), the average values of both $Q_{6}$ and $Q_{6}^{*}$ are shown as functions of the tap intensity $\Gamma$. Both $Q_{6}$ and $Q_{6}^{*}$ behave nonmonotonically in resemblance to the behavior of the packing fraction (Fig. 1). Nevertheless, some differences appear when the results are plotted as functions of the packing fraction [Figs. 4(c) and 4(d)]. When computed taking into account the actual contacts (i.e., $Q_{6}^{*}$ ), the bond order parameter suggests the existence of two branches: the highest (lowest) corresponds to the lowest (highest) values of $\Gamma$. This means that the bond order parameter might be the right descriptor to distinguish between states at each side of the minimum density. However, we cannot make a strong statement since the relative distance between the branches in $Q_{6}^{*}$ is only $1 \%$, at most, and the standard error is larger than this [see the error bars in Figs. 4(c) and 4(d)]. In experiments, this minute variation could render this measure useless as a method to distinguish steady states of equal density. Interestingly, the two branches collapse into a single curve if we compute the bond order parameter for nearest neighbors $Q_{6}$. This is an indication that descriptors based on contacts rather than purely geometrical arrangements are more suitable to distinguish between steady states with the same $\phi$ but obtained with different $\Gamma$.

We have run additional simulations for two different stiffnesses of the disks. The results for the bond order parameter are presented in Fig. 5 for disks with stiffness $2 k_{n}$ (stiff) and $k_{n} / 2$ (soft). The bond order parameters for nearest neighbors $\left(Q_{6}\right)$ and for contacts $\left(Q_{6}^{*}\right)$ as functions of tap intensity [Figs. 5(a) and 5(b)] follow the same general trends, the only difference being a displacement in the position of the minimum. This shift of the position of the minimum $Q_{6}$ is coincident with a shift in the position $\Gamma_{\min }$ of the minimum packing fraction. The shift is due to a change in the effective expansion of the granular column during a tap (this is the ultimate effect of the excitation through taps). Softer disks are more dissipative (have a lower 

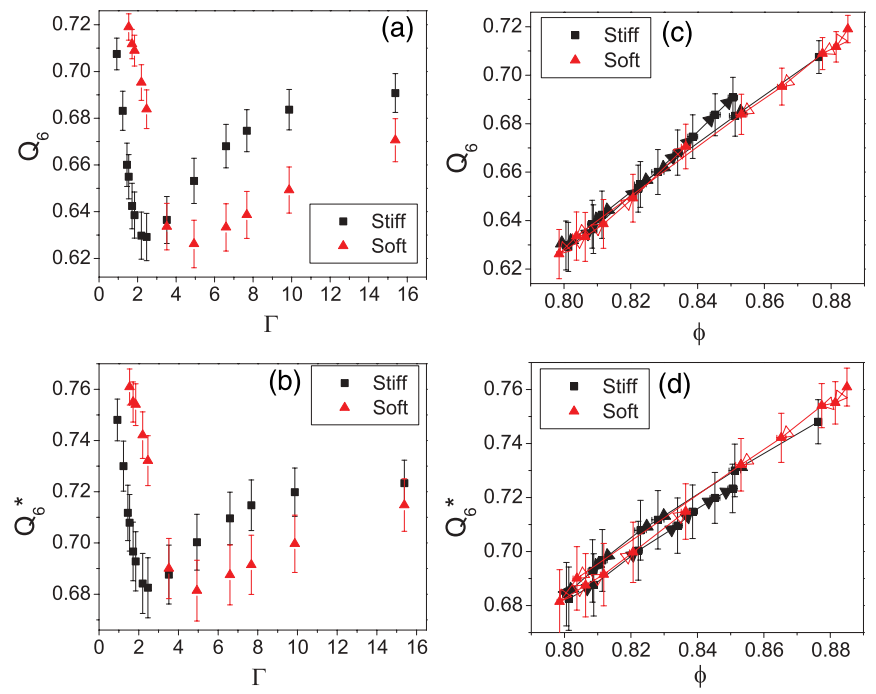

FIG. 5. (Color online) Average value of the bond order parameter for stiff $\left(2 k_{n}\right)$ and soft $\left(k_{n} / 2\right)$ disks; nearest-neighbors $Q_{6}$ (upper panel) and contact $Q_{6}^{*}$ (lower panel). (a) and (b) show the mean value of the bond order parameter as a function of the tap amplitude $\Gamma$ (squares for stiff and triangles for soft disks), and (c) and (d) as a function of the density $\phi$ (squares and closed arrows for stiff disks, and triangles and open arrows for soft disks). In (c) and (d), the arrows indicate the direction of increasing $\Gamma$.

restitution coefficient for given $\gamma_{n}$ ). Hence, softer disks require stronger taps for the granular bed to expand enough to reach the high-intensity regime where the packing fraction starts to grow with tap intensity. For both stiff and soft disks, when the bond order parameter is considered as a function of density [Figs. 5(c) and 5(d)], there is a narrow gap between the two branches for $Q_{6}^{*}$ which is not present if one considers $Q_{6}$, as we observed before. The qualitative behavior of the bond order parameters is therefore unaffected by the stiffness of the disks.

Since the bond order parameter generally presents a distribution with characteristic peaks for partially ordered systems, we now focus on this distribution and compare two equal-density pairs of states at each side of the minimum density to see if any clear difference can be observed. In Fig. 6, we show the distribution of $Q_{6}$ and $Q_{6}^{*}$ for these four states. Clearly, this parameter is a poor indicator for distinguishing between equal-density steady states. The two states at high density and the two at low density are fairly paired for both bond order parameters, especially in the region of intermediate values, and at the peaks. At high values $\left(Q_{6} \geqslant 0.75\right)$, the data from the high and low tap intensities seem to slightly separate (except at the peaks), yet it becomes difficult to infer a clear systematic trend.

In summary, the bond order parameters $Q_{6}$ and $Q_{6}^{*}$, the pair correlation function $g(r)$, and the size distribution of Voronoi cells are parameters that subtly suggest that steady states with the same $\phi$ but obtained with different tap intensities are, indeed, different. Nevertheless, the differences displayed are very small, which implies that none of these measurements can be univocally used to differentiate the states.
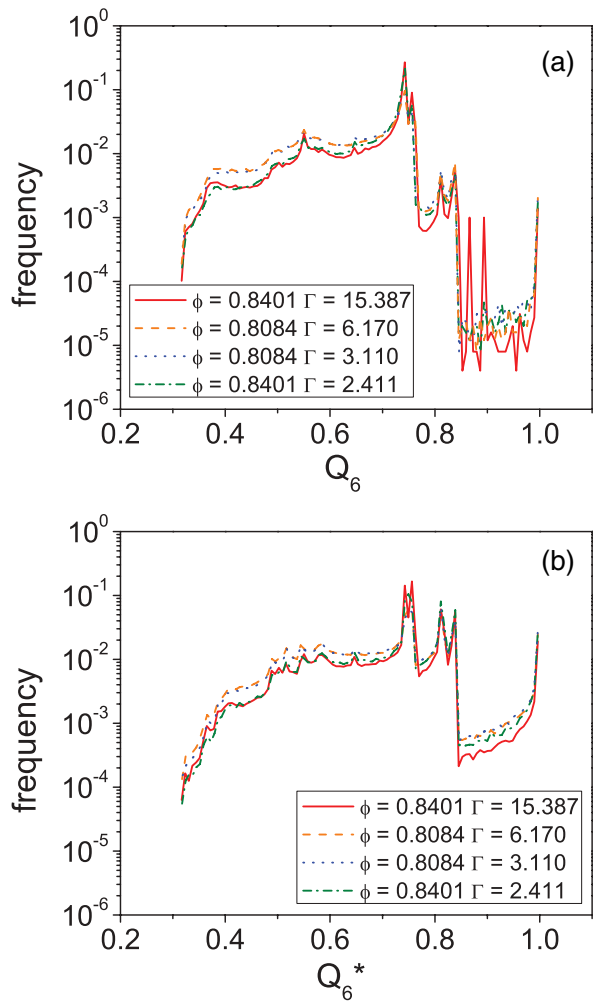

FIG. 6. (Color online) Distribution of $Q_{6}$ and $Q_{6}^{*}$ for the four states compared in the text. The signals appear paired for states of equal density, without any salient feature allowing one to distinguish between them.

\section{STRUCTURE OF THE CONTACT NETWORK}

As we mentioned in the Introduction, steady states of equal density at the two sides of the minimum density can be distinguished by the force moment tensor. Hence, it seems plausible that the properties of the contact network also allow such a distinction. Indeed, the contact network carries a portion of the information contained in the contact forces which is at the heart of the stress tensor definition. A result that supports this idea is that the bond order parameter based on actual contacts (i.e., $Q_{6}^{*}$ ) is more suitable to distinguish between states of the same $\phi$ than the $Q_{6}$ based on just first neighbors.

A natural approach to further explore the properties of the contact network is to define a graph considering every contact between particles as an edge and each grain as a node (Fig. 7). Every grain representing a node in the network is equilibrated by at least two contacts (edges), and the graph as a whole is responsible for the transmission of stresses through the packing [36]. Note that, due to gravity, in our samples there are no rattlers, i.e., particles without any contact. The graphs are then characterized by means of the following descriptors [37]: (a) the mean shortest distance (or mean topological distance) $\langle l\rangle$ between two given nodes measured by number of edges; (b) the diameter $D$ of the network, which is the largest topological distance in the graph; and (c) the degree of the network $\langle k\rangle$ which is equivalent to the average contact number per grain, $Z$. Furthermore, we analyze the networks by measuring the number of polygons: closed loops whose size is measured in the number of contacts (or edges) (Fig. 7). In the following, 


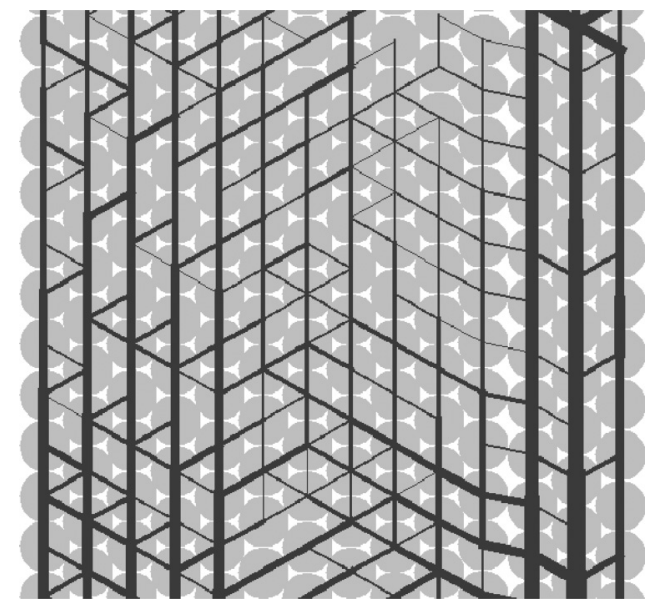

FIG. 7. Part of one of the deposits analyzed. In dark gray, edges joining the centers of grains in contact. The width of the edges is proportional to the corresponding normal force. Different loops (triangles, squares, pentagons, and hexagons) can be observed.

we term these empty closed loops simply as triangles, squares, pentagons, and so on.

The role of polygons was originally introduced in [38] where it was shown that odd loops are rigid due to the frustration of rotations, playing an important role in the rigidity of a granular sample. Following this argument, triangles-the minimal polygonal structure-were proposed as an order parameter to characterize the jamming transition in an isotropically compressed granular packing [36]. Indeed, while the number of triangles grows in the proximity of the jamming point, the population of the other loops remains constant or decreases [27]. This idea was also applied to characterize the rheological properties of sheared granular materials (see Ref. [25] and references therein), showing the usefulness of this parameter to characterize the evolution of the equilibrium states developed by a granular material under external loads.

In Figs. 8(a)-8(c), we show the results of $\langle l\rangle, D$, and $\langle k\rangle$ for different values of the tap intensity $\Gamma$. As with the packing fraction in Fig. 1, all these descriptors have a nonmonotonic dependence on $\Gamma$. For tap intensities below $\Gamma_{\min },\langle l\rangle$ and $D$ grow as $\Gamma$ is increased, indicating that the network is becoming more disconnected. Correspondingly, $\langle k\rangle$ decreases until the system reaches its minimum packing fraction around $\Gamma \simeq 4.0$. Beyond this point, the contact network recovers connections ( $\langle k\rangle$ increases), thus reducing $\langle l\rangle$ and $D$. The importance of these topological parameters in characterizing the spatial structure of the sample becomes more evident from Figs. 8(d)-8(f) where the values of $\langle l\rangle, D$, and $\langle k\rangle$ are presented as functions of $\phi$. In all these plots two branches are distinguishable, each corresponding to a different side of $\Gamma_{\min }$. This implies that the topological descriptors are able to distinguish between states with equal $\phi$ obtained at different tap intensities. However, care should be taken as the relative differences between different states with the same $\phi$ are very small. Indeed, the greatest relative difference is about $5 \%$ (for the case of $\langle k\rangle$ in the states with $\phi \simeq 0.84$ ). The fact that the resolution is very high in our numerical simulations becomes key to differentiating the states with the same $\phi$.
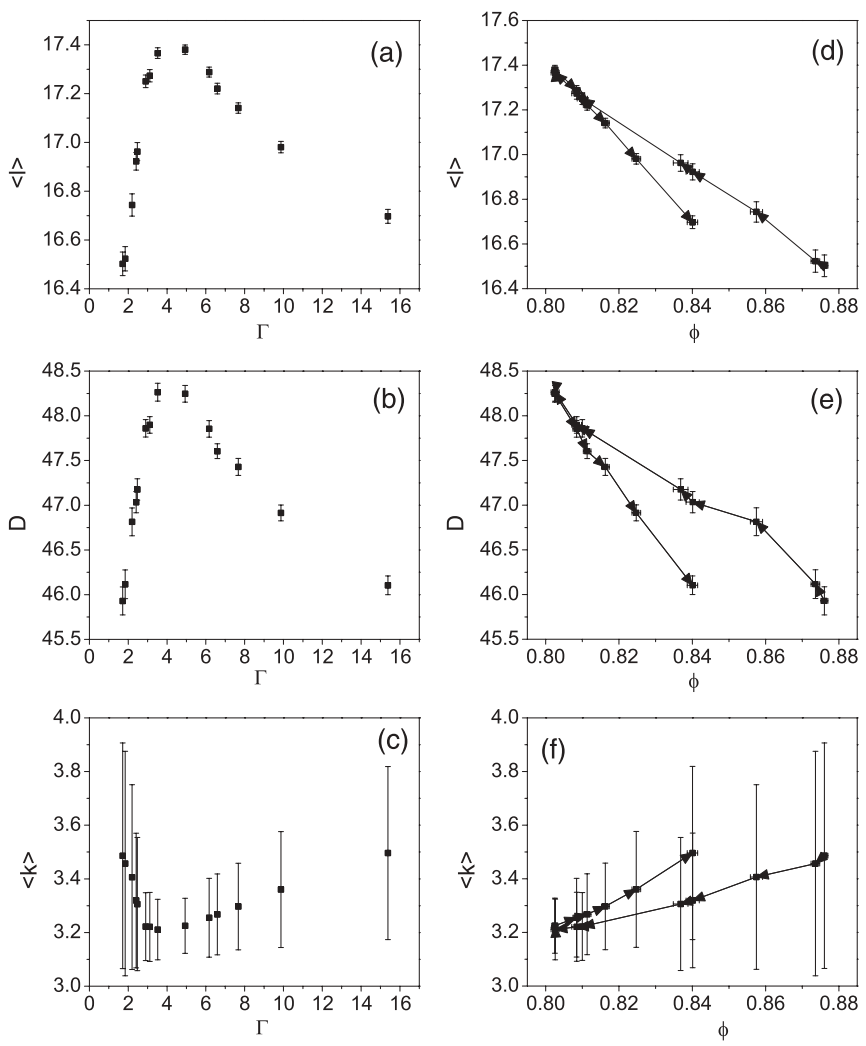

FIG. 8. Average shortest distance $\langle l\rangle$, average diameter $D$, and average connectivity $\langle k\rangle$ as functions of the tap intensity $\Gamma$ (a)-(c), and as functions of the mean packing fraction $\phi(d)-(f)$. The arrows indicate the direction of increasing $\Gamma$.

The number of loops (or polygons) in the network is shown in Fig. 9. As in Fig. 8, the left (right) column shows the average population of each type of motif as a function of the tap intensity (packing fraction). Figures 9(a)-9(e) reveal that, for low values of $\Gamma$, small polygons-triangles and squares-decrease in number as $\Gamma$ increases and the network becomes disordered and disconnected. Instead, big polygons - pentagons and heptagons-become more numerous. For $\Gamma>\Gamma_{\min }$ this tendency is reversed and the number of small (big) polygons increases (decreases) as the tap intensity is augmented. Notably, hexagons behave in a peculiar way; their population decreases monotonically with $\Gamma$. Although the decrease becomes attenuated for $\Gamma>\Gamma_{\min }$, the monotonic behavior of hexagons is a unique feature which has not been observed in any other descriptor-either geometrical or topological-considered so far. We speculate that this particular behavior of the number of hexagons is strongly related to the boundary effects. Most hexagons observed are located near the vertical walls of the container (as exemplified by Fig. 7). This phenomenon could be associated with the special columnar arrangements of particles induced by the walls [39-41]. However, we will not further pursue this issue in this paper.

The behavior of the number of polygons within the network can be easily linked to the results obtained for the other topological properties. Indeed, increasing the number of small polygons adds connectivity to the network. For example, if we consider a group of three nodes connected through two 

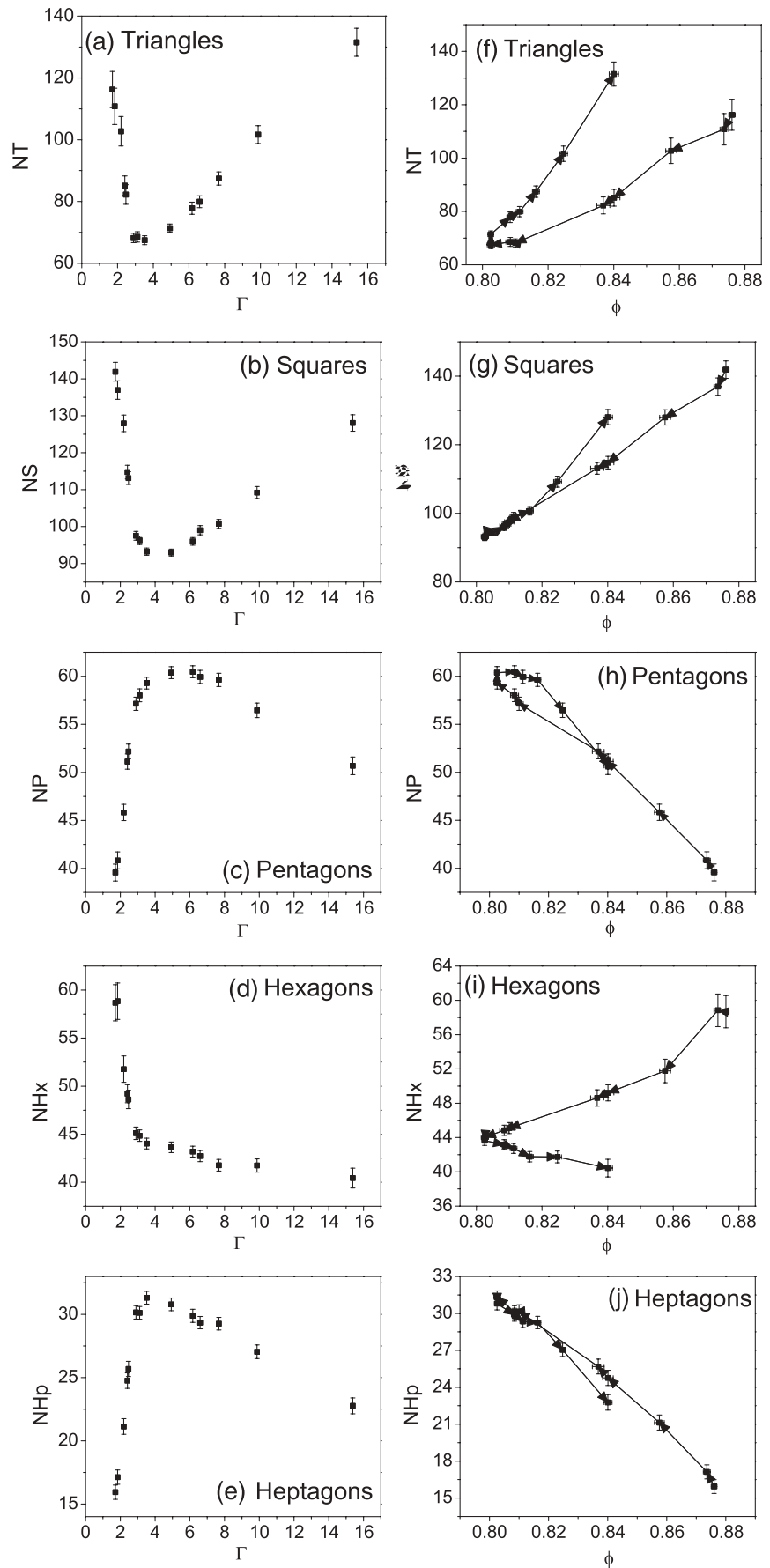

FIG. 9. The populations of polygons defined in the text as a function of the tap intensity (a)-(e), and as a function of the mean packing fraction (f)-(j). The arrows indicate the direction of increasing $\Gamma$. NT, NS, NP, NHx, and NHp indicate the numbers of triangles, squares, pentagons, hexagons, and heptagons, respectively.

edges, the distance is $\langle l\rangle=2$ and the average connectivity is $\langle k\rangle=4 / 3$. If a triangle is formed by adding one edge, the distance reduces to $\langle l\rangle=1$ and the connectivity rises to $\langle k\rangle=2$. For $\Gamma<\Gamma_{\min }$, increasing $\Gamma$ leads to a reduction in the number of small polygons and, hence, the network connectivity is reduced. For $\Gamma>\Gamma_{\min }$, the behavior reverses, with large polygons being divided into smaller ones by new edges.
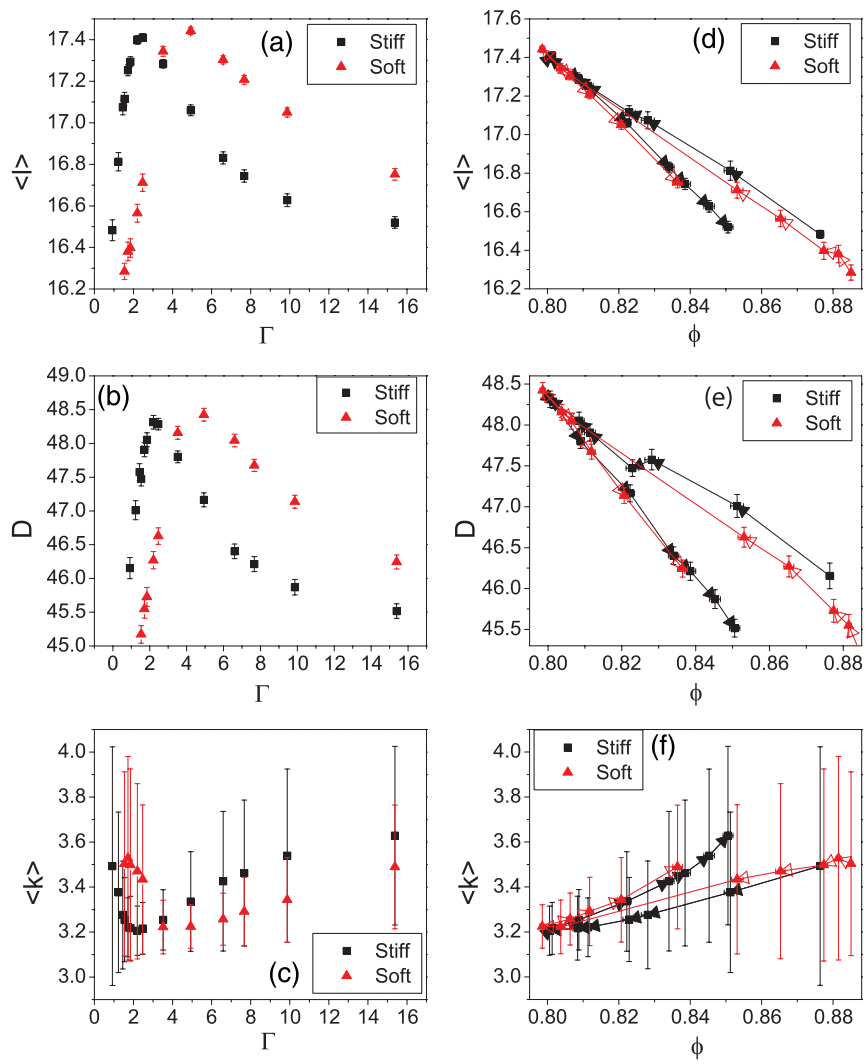

FIG. 10. (Color online) Average shortest distance $\langle l\rangle$, average diameter $D$, and average connectivity $\langle k\rangle$ for stiff (squares) and soft (triangles) disks, as functions of the tap intensity $\Gamma$ (a)-(c), and as functions of the mean packing fraction $\phi(d)-(f)$. The arrows (closed for stiff disks, open for soft disks) indicate the direction of increasing $\Gamma$.

The population of polygons as a function of the density [see Figs. 9(f)-9(j)] reveals - like the other topological descriptors - the existence of two branches, one at each side of the minimum. This behavior is especially clear for the cases of triangles and hexagons. In brief, we can state that the branch of higher $\Gamma$ is characterized by a higher number of triangles and a lower number of hexagons, in comparison with the branch of lower $\Gamma$.

We present the results of the topological measurements for stiff and soft disks in Figs. 10 and 11. In Figs. 10(a)-10(c) the shortest distance $\langle l\rangle$, the diameter $D$, and the connectivity $\langle k\rangle$ of the networks are plotted as functions of the tap intensity. Figures 10(d)-10(f) display the same results as functions of the density. As we can observe, despite the shift in $\Gamma$, all the conclusions drawn for the original stiffness are valid also for softer and stiffer disks.

In Fig. 11 we show the results obtained for the populations of polygons, as functions of the tap intensity [Figs. 11(a)11(e)] and as functions of the density [Figs. 11(f)-11(j)]. The observations highlighted previously remain unchanged for the different stiffnesses. Hexagons remain the only polygons whose population is monotonic in $\Gamma$. The two branches in Figs. 11(f)-11(j) appear well separated only for triangles and hexagons. The loop in the population of pentagons remains present. Interestingly, although the branches in the population 

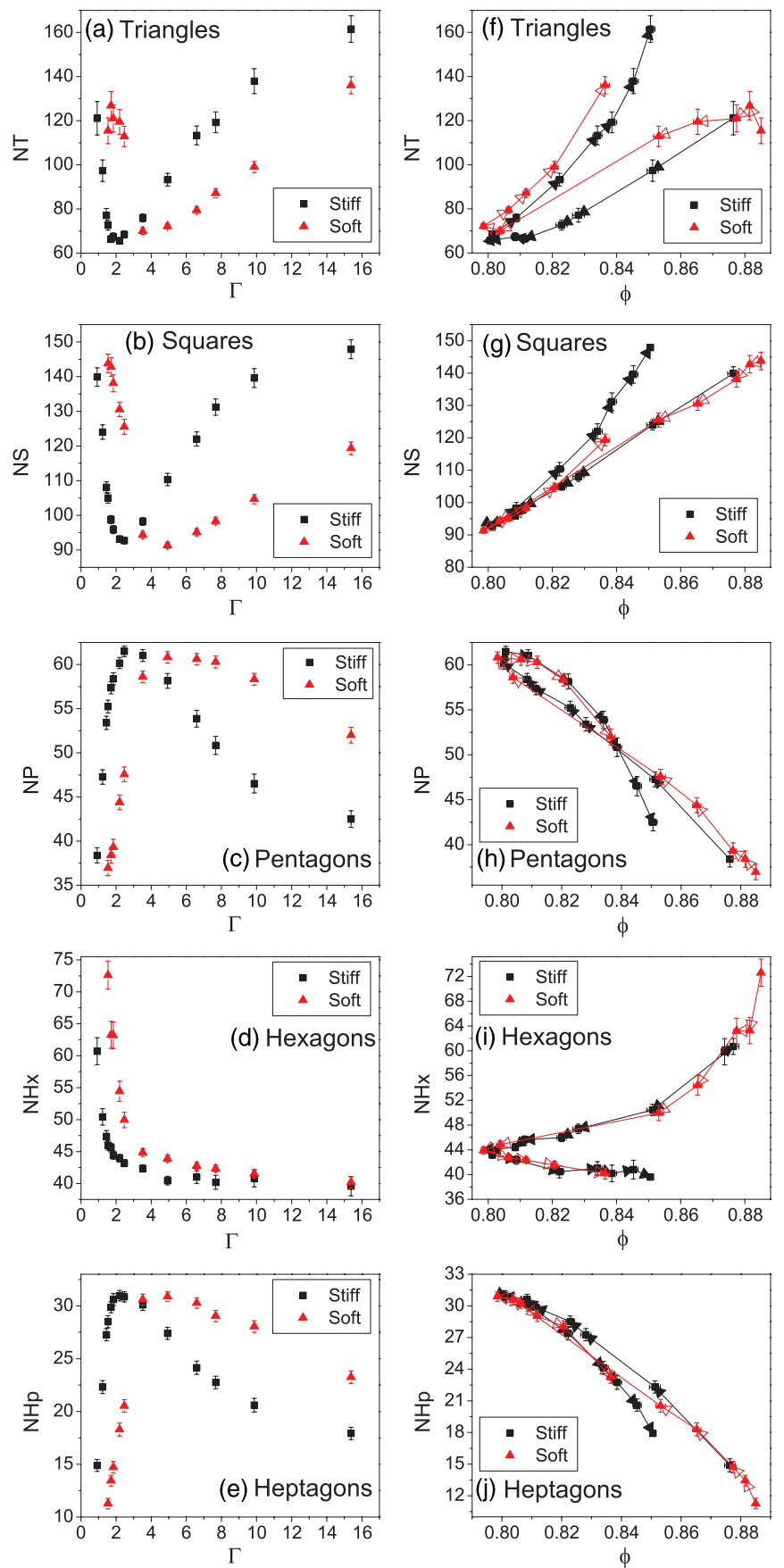

FIG. 11. (Color online) The populations of polygons for stiff (squares) and soft (triangles) disks as a function of the tap intensity (a)-(e), and as a function of the mean packing fraction (f)-(j). The arrows (closed for stiff disks, open for soft disks) indicate the direction of increasing $\Gamma$.

of heptagons are rather collapsed for stiffnesses $k_{n} / 2$ and $k_{n}$, a slight separation appears for the stiffest disks considered.

At this point, we may wonder which is the best topological indicator to distinguish between two different states with the same packing fraction. In order to answer this question, we compare the two pairs of steady states of equal $\phi$ at each side of the minimum density that were analyzed in Sec. III. The topological indicators on these states are summarized in Table I for the pair $A$ of steady states obtained with high density,
TABLE I. Topological descriptors for two states of equal, high packing fraction.

\begin{tabular}{lrrr}
\hline \hline$\Gamma$ & 2.411 & 15.387 & Relative \\
$\phi$ & 0.840101 & 0.840053 & \begin{tabular}{c} 
change \\
\hline$\langle l\rangle$
\end{tabular} \\
$D$ & 16.923 & 16.697 & $-2 \%$ \\
$\langle k\rangle$ & 47.034 & 46.104 & $-2 \%$ \\
$\mathrm{NT}$ & 3.319 & 3.496 & $+5 \%$ \\
$\mathrm{NS}$ & 85.122 & 131.510 & $+54 \%$ \\
$\mathrm{NP}$ & 114.758 & 128.046 & $+12 \%$ \\
$\mathrm{NH}$ & 51.128 & 50.686 & \\
$\mathrm{NHp}$ & 49.218 & 40.430 & $-18 \%$ \\
\hline \hline
\end{tabular}

and Table II for the pair $B$ of steady states obtained with low density. Focusing first on the pair with high density, we see that the relative variation in $\langle l\rangle$ and $D$ is about $-2 \%$ (the state obtained at higher $\Gamma$ takes smaller values than the one obtained at lower $\Gamma$ ). In accordance, the average connectivity for the state at higher $\Gamma$ increases by around $5 \%$. The population of polygons exhibits more significant variations: the number of triangles rises by $54 \%$, and that of squares by $12 \%$. At the same time, the number of hexagons decreases by $18 \%$ and that of heptagons decreases by $8 \%$. The population of pentagons seems to remain fairly constant with a decrease of less than $1 \%$. These important changes in the population of polygons that just imply minute changes in the connectivity can be understood as follows. Converting a hexagon into four triangles requires the introduction of three new links in the network (Fig. 7). Then, 9 hexagons may give rise to 36 triangles by just adding 27 links which indeed implies an increase of $\langle k\rangle$ of around 0.1 (recall that the network is composed of 512 particles). Naturally, this is the simplest combination we can think of. Hexagons can also give rise to squares, and we see that the number of heptagons slightly decreases, thus contributing also to the rise the populations of small polygons. In summary, the important observation is that a slight change in the connectivity of the network leads to dramatic changes in the number of polygons, without having an impact on the density of the packing.

The differences in the pair $B$ of steady states with low density (Table II) show the same qualitative behavior, although the relative changes are smaller due to the proximity of the values of $\Gamma$ used to obtain them. Again, the largest variations

TABLE II. Topological descriptors for two states of equal, low packing fraction.

\begin{tabular}{lccc}
\hline \hline$\Gamma$ & 3.110 & 6.170 & \\
$\phi$ & 0.808458 & 0.808433 & $\begin{array}{c}\text { Relative } \\
\text { change }\end{array}$ \\
\hline$\langle l\rangle$ & 17.273 & 17.288 & \\
$D$ & 47.898 & 47.854 & $+1 \%$ \\
$\langle k\rangle$ & 3.221 & 3.255 & $+13 \%$ \\
NT & 68.546 & 77.826 & $+4 \%$ \\
NS & 96.282 & 96.012 & $-4 \%$ \\
NP & 58.024 & 60.468 & \\
NH & 44.850 & 43.188 & \\
NHp & 30.122 & 29.89 & \\
\hline \hline
\end{tabular}


are observed in the populations of triangles and hexagons, with an increase of $13 \%$ for the former and a decrease of $4 \%$ for the latter. Thus, the population of polygons seems to be a suitable topological property, as it is more sensitive than other measurements to changes in the structure of the packing or-as in this case - to distinguish between different states of mechanical equilibrium.

\section{CONCLUSIONS}

We have studied the contact network structure of the steady states reached by a granular layer when submitted to vertical tapping. It has been recently shown [6] that the volume fraction of the system is not a monotonic function of the tap amplitude and that an extra variable is necessary to univocally identify each steady state of the system [7].

In this work, we have shown that standard geometrical measurements such as the pair correlation function or the size distribution of Voronoi cells are unable to give a clear distinction between states of equal packing fraction that are known to differ in the average moment tensor. Thus, we have implemented a series of topological measurements based on the contact network of the resulting packings. This approach allows us to distinguish between such different states.

We can understand the suitability of a topological approach over any geometrical measurement based on the fact that minute changes in the actual position of a single particle can induce dramatic variations in the contact network as a whole. The topology of the interparticle contacts univocally deter- mines the stress transmission of each mechanical equilibrium state, but may not affect, in a distinguishable way, the density or the number of nearest neighbors (defined by proximity rather than contact). The contact network, instead, will reflect such small changes, thus becoming a more sensitive probe of the state of the system.

The family of polygonal structures displayed by the contact network may be thought of as a mesoscopic extension of the geometrical measurements. Effectively, the bond order parameters and Voronoi tessellations are highly local metrics based on information about the nearest neighbors, regardless of whether there is actually a contact or not. The polygons are based on contacts but also extend and consider the neighbors of neighbors. This mesoscopic nature allows polygons to capture information lost in a purely local context and could be considered as a suitable scale to define a representative volume element in order to perform a homogenization process of the system as a whole.

\section{ACKNOWLEDGMENTS}

R.A. thanks MIUR-FIRB RBFR081IUK for financial support. L.A.P. acknowledges The Aspen Center for Physics and Instituto de Física de Líquidos y Sistemas Biológicos (CONICET La Plata, UNLP) where parts of this work were done. I.Z. and D.M. acknowledge Grant No. FIS2011-26675 (Spanish Government) and PIUNA (Universidad de Navarra) for their support.
[1] E. R. Nowak, J. B. Knight, E. Ben-Naim, H. M. Jaeger, and S. R. Nagel, Phys. Rev. E 57, 1971 (1998).

[2] E. R. Nowak, J. B. Knight, M. L. Povinelli, H. M. Jaeger, and S. R. Nagel, Powder Technol. 94, 79 (1997).

[3] Ph. Ribière, P. Richard, P. Philippe, D. Bideau, and R. Delannay, Eur. Phys. J. E 22, 249 (2007).

[4] M. Schröter, D. I. Goldman, and H. L. Swinney, Phys. Rev. E 71, 030301(R) (2005).

[5] M. Pica Ciamarra, A. Coniglio, and M. Nicodemi, Phys. Rev. Lett. 97, 158001 (2006).

[6] L. A. Pugnaloni, I. Sánchez, P. A. Gago, J. Damas, I. Zuriguel, and D. Maza, Phys. Rev. E 82, 050301(R) (2010).

[7] L. A. Pugnaloni, J. Damas, I. Zuriguel, and D. Maza, Pap. Phys. 3, 030004 (2011).

[8] S. F. Edwards and R. B. S. Oakeshott, Physica A 157, 1080 (1989).

[9] S. F. Edwards and C. C. Mounfield, Physica A 226, 1 (1996).

[10] A. Mehta and S. F. Edwards, Physica A 157, 1091 (1989).

[11] S. F. Edwards and D. V. Grinev, Phys. Rev. E 58, 4758 (1998).

[12] S. F. Edwards and D. V. Grinev, Granular Matter 4, 147 (2003).

[13] J. H. Snoeijer, T. J. H. Vlugt, W. G. Ellenbroek, M. van Hecke, and J. M. J. van Leeuwen, Phys. Rev. E 70, 061306 (2004).

[14] S. F. Edwards, Physica A 353, 114 (2005).

[15] S. Henkes, C. S. OHern, and B. Chakraborty, Phys. Rev. Lett. 99, 038002 (2007).

[16] S. Henkes and B. Chakraborty, Phys. Rev. E 79, 061301 (2009).
[17] R. Blumenfeld and S. F. Edwards, J. Phys. Chem. B 113, 3981 (2009).

[18] B. P. Tighe, A. R. T. van Eerd, and T. J. H. Vlugt, Phys. Rev. Lett. 100, 238001 (2008).

[19] P. A. Gago, N. E. Bueno, and L. A. Pugnaloni, Granular Matter 11, 365 (2009).

[20] L. A. Pugnaloni, M. Mizrahi, C. M. Carlevaro, and F. Vericat, Phys. Rev. E 78, 051305 (2008).

[21] M. E. J. Newman, SIAM Rev. 45, 167 (2003).

[22] J. Berg and A. Mehta, Europhys. Lett. 56, 784 (2001).

[23] J. A. Dodds, J. Colloid Interface Sci. 77, 317 (1980).

[24] S. Ostojic, E. Somfai, and B. Nienhuis, Nature (London) 439, 828 (2006).

[25] D. M. Walker and A. Tordesillas, Phys. Rev. E 85, 011304 (2012).

[26] D. S. Bassett, E. T. Owens, K. E. Daniels, and M. A. Porter, Phys. Rev. E 86, 041306 (2012).

[27] R. Arévalo, I. Zuriguel, and D. Maza, Phys. Rev. E 81, 041302 (2010).

[28] P. A. Cundall and O. D. L. Strack, Geotechnique 29, 47 (1979).

[29] R. Arévalo, D. Maza, and L. A. Pugnaloni, Phys. Rev. E 74, 021303 (2006).

[30] F. Ludewig, S. Dorbolo, T. Gilet, and N. Vandewalle, Europhys. Lett. 84, 44001 (2008).

[31] S. Torquato, Random Heterogeneous Material (Springer, New York, 2002).

[32] J. S. Olafsen and J. S. Urbach, Phys. Lett. 95, 098002 (2005). 
[33] T. Aste, T. D. Matteo, M. Saadatfar, T. J. Senden, M. Schröter, and H. L. Swinney, Europhys. Lett. 79, 24003 (2007).

[34] P. J. Steinhardt, D. R. Nelson, and M. Ronchetti, Phys. Rev. B 28, 784 (1983).

[35] P. Richard, L. Oger, J. P. Troadec, and A. Gervois, Phys. Rev. E 60, 4551 (1999).

[36] R. Arévalo, I. Zuriguel, S. Ardanza-Trevijano, and D. Maza, Int J. Bifurcation Chaos Appl. Sci. Eng. 20, 897 (2010).
[37] R. Arévalo, I. Zuriguel, and D. Maza, Int. J. Bifurcation Chaos Appl. Sci. Eng. 19, 695 (2009).

[38] N. Rivier, J. Non-Cryst. Solids 352, 42 (2006).

[39] I. Bartos and I. M. Janosi, Granular Matter 9, 81 (2007).

[40] I. Zuriguel, T. Mullin, and R. Arévalo, Phys. Rev. E 77, 061307 (2008).

[41] H. K. Chan, Phys. Rev. E 84, 050302(R) (2011). 